\title{
Editorial
}

\section{Multiscale Discrete Simulation of Complex Systems}

\author{
Qingang Xiong, ${ }^{1}$ Jingsen $\mathrm{Ma}^{2}{ }^{\mathrm{Hao}} \mathrm{Zhou}^{3}$ and Giulio Lorenzini ${ }^{4}$ \\ ${ }^{1}$ Computer Science and Mathematics Division, Oak Ridge National Laboratory, Oak Ridge, TN 37831, USA \\ ${ }^{2}$ Dynaflow Inc., Jessup, MD 20794, USA \\ ${ }^{3}$ Institute for Thermal Power Engineering, Zhejiang University, Hangzhou 310027, China \\ ${ }^{4}$ Department of Industrial Engineering, University of Parma, 43124 Parma, Italy
}

Correspondence should be addressed to Qingang Xiong; xiongq@ornl.gov and Jingsen Ma; Jingsen Ma; jingsen@dynaflow-inc.com

Received 12 May 2015; Accepted 12 May 2015

Copyright (C) 2015 Qingang Xiong et al. This is an open access article distributed under the Creative Commons Attribution License, which permits unrestricted use, distribution, and reproduction in any medium, provided the original work is properly cited.

Complex systems exist in many aspects of nature and society and affect our daily life significantly. Computational modeling is a powerful tool to complement theories and experiments for leveraging the knowledge on the fundamental mechanisms in complex systems. Discrete methods account for a large portion in the modeling of complex systems, whose advantages over traditional continuum methods are, for example, mathematical concision, easy implementation, and high parallelization. Thus, it is highly necessary to track the latest progress on the discrete simulation of complex systems. This special issue consists of papers on the development and application of discrete approaches in modeling of complex systems. Although the selected papers in this special issue cannot represent all aspects of the discrete simulation of complex systems, they stand at the frontier of the area and we are pleased to share these valuable papers with readers.

In the paper entitled "Dynamic Modeling and Optimal Control for Complex Systems with Statistical Trajectory” L. Guo et al. proposed a novel discrete-time nonlinear working state class dynamic mathematical model through $K$-means cluster method for sintering machines. Based on the working state class dynamic modeling, an optimal control method was developed to track trajectory for sintering machines. The proposed optimal control method for trajectory tracking was proved to be effective.

In the paper entitled "Algorithms for Routing Optimization in Multipoint to Multipoint 4PL System" J. Li et al. studied the multipoint to multipoint $4 \mathrm{PL}$ system routing optimization taking into account reliability constraint and objective factors. The established mathematical model for the system routing optimization was solved by the so-called messy genetic algorithm. Experimental results were used to validate the optimization algorithm.

In the paper entitled "System Dynamics Simulation of Large-Scale Generation System for Designing Wind Power Policy in China" L. Hou focused on the renewable energy policy in China, especially about the wind power. A system dynamics model was built to simulate and compare the wind power generation policies and a scenario analysis was performed to compare their effectiveness.

In the paper entitled "Research on the Development Approach for Reusable Model in Parallel Discrete Event Simulation" the model reuse in parallel discrete event simulation was investigated by J. Li et al. A model description which completes reusable component model framework (RCMF) was represented through their development tool-SuKit. Along with a model-customized description file with the configured information, SuKit is able to generate a skeleton RCMF model. Case study indicates that SuKit has good capability of developing RCMF models and the well-formed description file is well suited to be used for model reuse and integration.

In the paper entitled "Forecasting Beijing Transportation Hub Areas's Pedestrian Flow Using Modular Neural Network" S. Wang et al. studied the issue of forecasting Beijing transportation hub areas' pedestrian flow by a modular neural network approach. More than thirty typical sidewalks in Beijing transportation hub areas were selected to obtain about 2200 effective data samples. A correlation analysis was conducted to analyze the relationship between pedestrian 
flow and its influential factors and a forecasting model was built. The forecasting modular neural network model was tested with good predictive capability.

In the paper entitled "Cognitive Emotional Regulation Model in Human-Robot Interaction" X. Liu et al. investigated the human intelligence for human-robot interaction through the cognitive emotional regulation model. In their study, robot's emotional state was controlled and determined by the cognitive energy. The model was verified by an emotional robot with different degrees of freedom and kinds of facial expressions. Verification indicates that robot with cognitive emotional regulation model performs more intelligently.

In the paper entitled "Automation Countermeasure System for Intersection Optimization" the method of automation countermeasure system intersection optimization was investigated by $\mathrm{H}$. Lu et al. based on a multiobjective programming model. The efficiency and applicability of the proposed modeling and computing methods were tested based on realworld intersection in Beijing, China.

In the paper entitled "Stability Analysis and Design of a Nonlinear Controller for Hot Rolling Coiler" R. Li et al. studied the algorithm about differential geometry design nonlinear controller. Under the rotating orthogonal coordinate system condition, the fifth nonlinear motor model was employed as the control benchmark. It was shown by the experimental results that the algorithm is suitable for highly order tracking control system with time-varying parameters.

\section{Acknowledgments}

All paper contributors are gratefully acknowledged for their excellent studies and graceful writing. Reviewers are also greatly appreciated for their kind and useful comments and suggestions which are indispensable to the success of this special issue.

Qingang Xiong Jingsen Ma

Hao Zhou

Giulio Lorenzini 


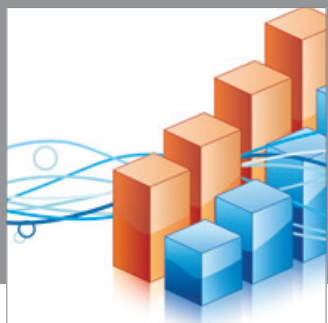

Advances in

Operations Research

mansans

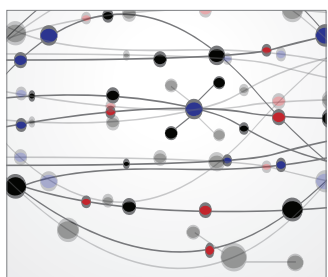

The Scientific World Journal
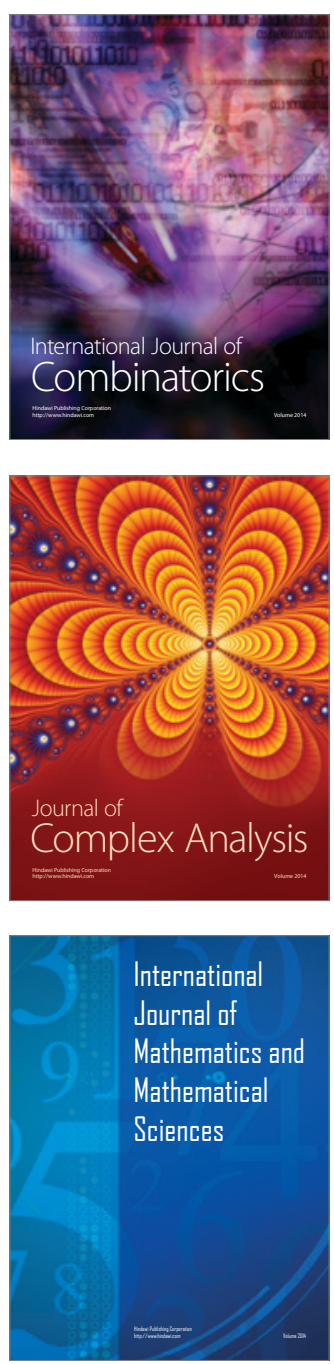
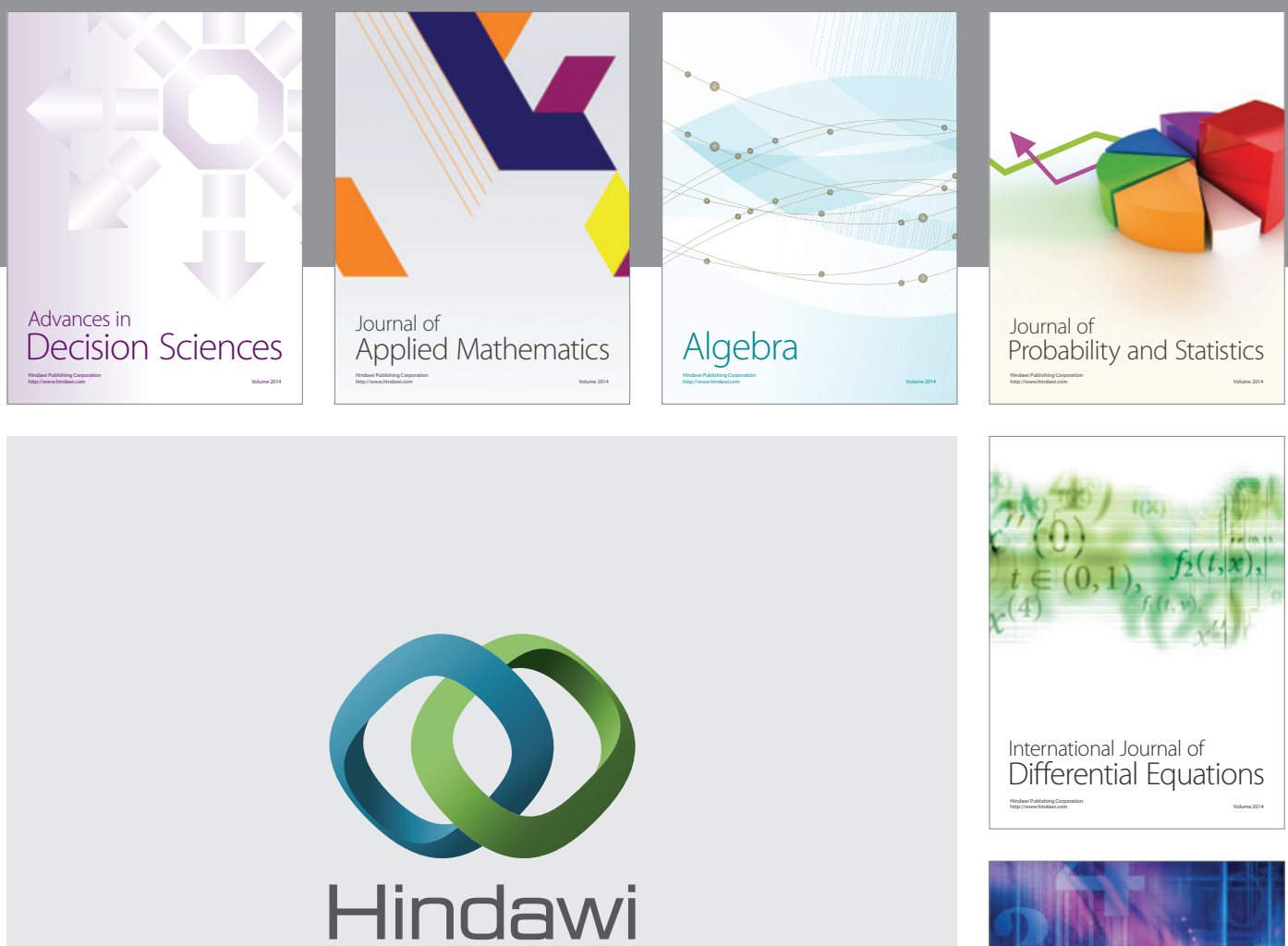

Submit your manuscripts at http://www.hindawi.com
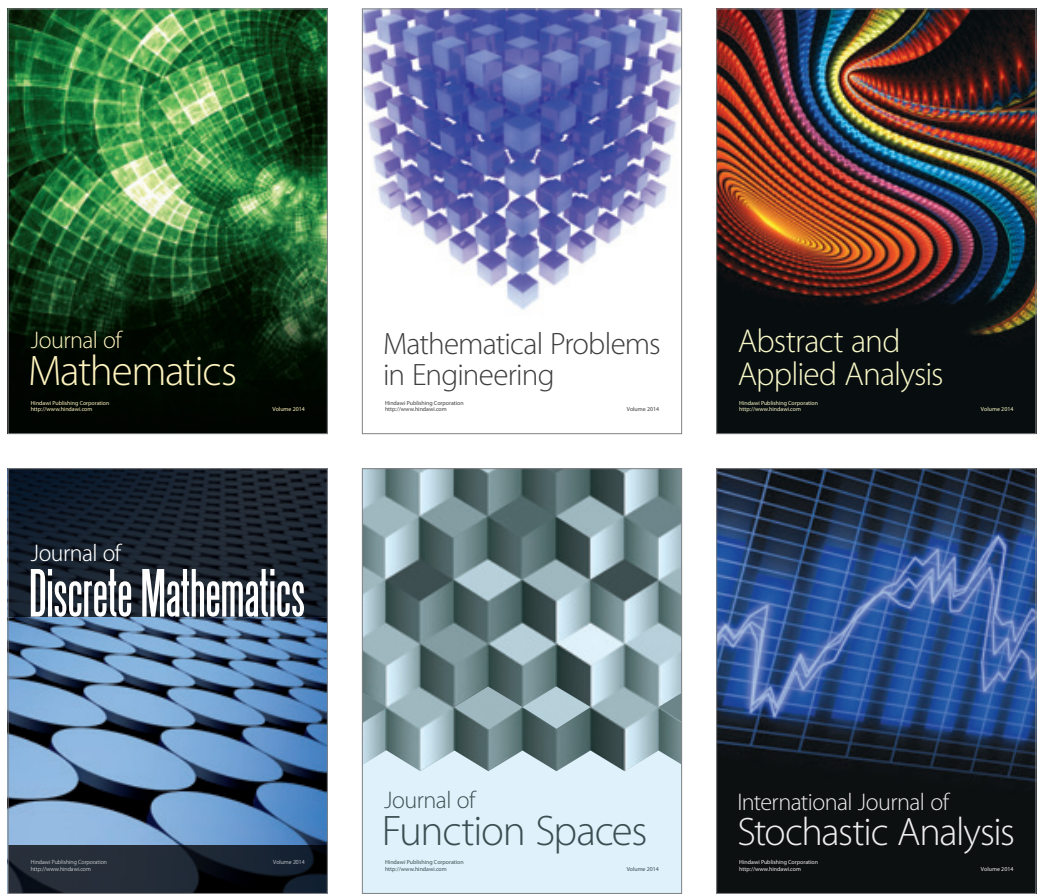

Journal of

Function Spaces

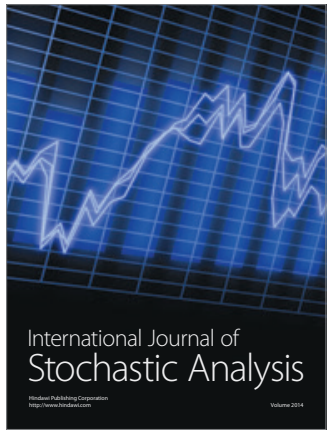

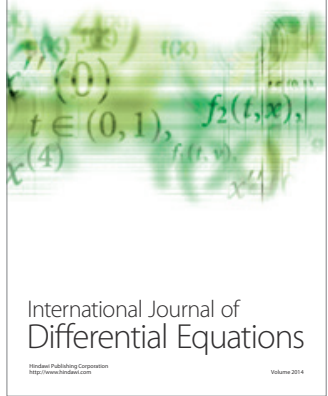
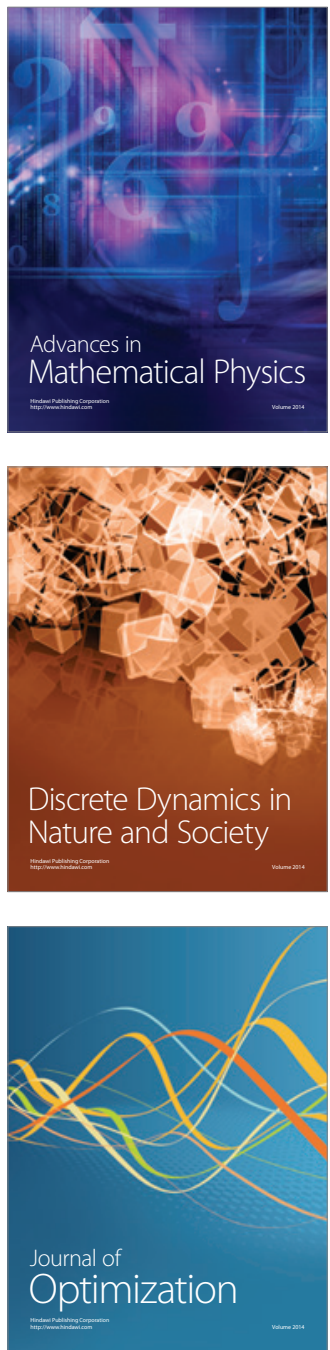